\title{
Phosphorylation of human Sgo1 by NEK2A is essential for chromosome congression in mitosis
}

\author{
Guosheng Fu $^{1}$, Xia Ding ${ }^{2,3}$, Kai Yuan ${ }^{1,2}$, Felix Aikhionbare ${ }^{2}$, Jianhui Yao $^{1}$, Xin Cai ${ }^{1,2}$, Kai Jiang ${ }^{1,2}$, Xuebiao Yao ${ }^{1,2}$ \\ ${ }^{1}$ Laboratory of Cellular Dynamics, Hefei National Laboratory, University of Science \& Technology of China, Hefei 230027, China; \\ ${ }^{2}$ Department of Physiology and Cancer Biology Program, Morehouse School of Medicine, Atlanta, GA 30310, USA, ${ }^{3}$ Department of \\ Medicine, Beijing University of Chinese Medicine, Beijing 100029, China
}

Chromosome segregation in mitosis is orchestrated by the interaction of the kinetochore with spindle microtubules. Our recent study shows that NEK2A interacts with MAD1 at the kinetochore and possibly functions as a novel integrator of spindle checkpoint signaling. However, it is unclear how NEK2A regulates kinetochore-microtubule attachment in mitosis. Here we show that NEK2A phosphorylates human Sgo1 and such phosphorylation is essential for faithful chromosome congression in mitosis. NEK2A binds directly to HsSgol in vitro and co-distributes with HsSgol to the kinetochore of mitotic cells. Our in vitro phosphorylation experiment demonstrated that HsSgol is a substrate of NEK2A and the phosphorylation sites were mapped to $\mathrm{Ser}^{14}$ and $\mathrm{Ser}^{507}$ as judged by the incorporation of ${ }^{32} \mathrm{P}$. Although such phosphorylation is not required for assembly of $\mathrm{HsSgol}$ to the kinetochore, expression of non-phosphorylatable mutant HsSgol perturbed chromosome congression and resulted in a dramatic increase in microtubule attachment errors, including syntelic and monotelic attachments. These findings reveal a key role for the NEK2A-mediated phosphorylation of HsSgol in orchestrating dynamic kinetochore-microtubule interaction. We propose that NEK2A-mediated phosphorylation of human Sgo 1 provides a link between centromeric cohesion and spindle microtubule attachment at the kinetochores.

Keywords: Sgo1, NEK2A, phosphorylation, microtubule, kinetochore

Cell Research (2007) 17:608-618. doi: 10.1038/cr.2007.55; publication online 10 July 2007

\section{Introduction}

Chromosome movements during mitosis are governed by the interaction of spindle microtubules with a specialized chromosome domain located within the centromere. This specialized region, called the kinetochore (e.g. Ref [1]), is the site for spindle microtubule-centromere association. In addition to providing a physical link between chromosomes and spindle microtubules, the kinetochore plays an active role in chromosomal segregation through microtubule motors and spindle checkpoint sensors located at or near it [2-4]. Several elegant studies demonstrate an essential role for the Shugoshin (Sgo) family of proteins in temporal control of centromeric separation in eukaryotic cells

Correspondence: Xuebiao Yao

Tel/Fax: +86 5513606304

E-mail: yaoxb@ustc.edu.cn

Received 9 May 2007; revised 11 May 2007; accepted 14 May 2007; published online 10 July 2007
[5-10]. Interestingly, vertebrate Sgo1 interacts strongly with microtubules in vitro and regulates kinetochore microtubule stability in vivo [6]. Thus, Sgol provides a novel link between sister centromere cohesion and microtubule interactions at kinetochores. However, it has remained elusive as to the precise function and regulation of Sgo1 in kinetochore-microtubule association.

Mitosis is orchestrated by signaling cascades that coordinate mitotic processes and ensure accurate chromosome segregation. The key switch for the onset of mitosis is the archetypal cyclin-dependent kinase, $\mathrm{Cdc} 2$. In addition to the master mitotic kinase $\mathrm{Cdc} 2$, mitosis also involves the actions of three other protein serine/threonine kinase families, the Polo kinases, Aurora kinases, and the NIMA-related kinases (NEK) [11, 12]. The latter family has proven the most enigmatic in function, although recent advances from several sources are beginning to reveal a common functional theme. Our recent study demonstrates that NEK2A is a kinetochore-associated protein kinase essential for faithful chromosome segregation [13]. However, it remains 
unknown how NEK2A governs dynamic kinetochore-microtubule association.

To delineate the molecular regulation of NEK2A in kinetochore-microtubule interaction, we immobilized recombinant kinetochore proteins onto a nitrocellulous membrane for assessing NEK2A-binding activity, which led to the identification of an inter-relationship between NEK2A and HsSgo1. Our biochemical characterization demonstrates that HsSgol is a novel substrate of NEK2A. Most importantly, the phosphorylation of HsSgol by NEK2A is essential for faithful chromosome congression and the proper attachment of spindle microtubule to the kinetochore. We propose that NEK2A-mediated phosphorylation of HsSgol links kinetochore-microtubule attachment to chromosome segregation dynamics.

\section{Materials and Methods}

\section{DNA construction}

Full-length human Sgol gene (NM_001012409) was generated by PCR from a human Testis cDNA library (Clontech) and cloned into T-vector (Takara Biotechnology, Dalian, China). To construct GFPHsSgo1, GST-HsSgol and FLAG-HsSgol plasmids, the full-length HsSgol cDNA was subcloned into pEGFP-C3 vector (Clontech, Palo Alto, CA, USA) digested with PstI and BamHI, pGEX-6P-1 vector (Amersham Biosciences Corp., Piscataway, NJ, USA) digested with BamHI and SalI, and p3×FLAG-myc-CMV-24 vector (Sigma) digested with HindIII and BamHI. GFP-tagged and GST-

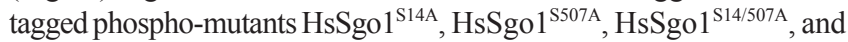
$\mathrm{HsSgol}^{\text {S14/507D }}$ were created by standard PCR methods with a sitedirected mutagenesis kit (Takara Biotechnology, Dalian, China). All constructs were sequenced in full.

\section{Reagents}

Monoclonal antibody against GFP was purchased from CLONTECH (Palo Alto, CA, USA); FLAG antibody (M2) and tubulin antibody were obtained from Sigma Chemical (St Louis, MO, USA); SGO1L1 monoclonal antibody was ordered from Abnova Inc. (Taiwan); and anti-NEK2A antibody was purchased from BD Biosciences (La Jolla, CA, USA). Recombinant active NEK2 kinase was purchased from Upstate Biotechnology.

\section{In vitro phosphorylation of HsSgol by NEK2A}

The GST-tagged wild-type HsSgol and non-phosphorylatable $\left(\mathrm{HsSgo1}^{\mathrm{S} 14 \mathrm{~A}}\right.$ HsSgo $^{\mathrm{S} 507 \mathrm{~A}}$ and HsSgo $\left.{ }^{\mathrm{S} 14 / 507 \mathrm{~A}}\right)$ mutants were expressed in Escherichia coli strain BL21 (DE3) and purified by using glutathione-agarose beads (Sigma) as previously described [13]. Briefly, 1 liter of LB media was inoculated with bacteria transformed with GSTHsSgol. The expression of protein was induced by addition of 0.5 $\mathrm{mM}$ isopropyl- $\beta$-D-thiogalactopyranoside at $30^{\circ} \mathrm{C}$ for $3 \mathrm{~h}$. After the induction, bacteria were harvested by centrifugation andre-suspended in phosphate-buffered saline (PBS) containing proteinase inhibitors (leupeptin, pepstatin, and chymostatin; $5 \mu \mathrm{g} / \mathrm{ml}$ ), and sonicated for four bursts of $10 \mathrm{~s}$ each by using a probe-tip sonicator. The lysis solution was cleared by centrifugation for $20 \mathrm{~min}$ at $10000 \times \mathrm{g}$. The soluble fraction was applied to a column packed with glutathioneagarose beads, followed by extensive washes with PBS.
For in vitro phosphorylation assay, aliquots of wild-type GSTHsSgol and mutant HsSgol proteins (20 $\mu \mathrm{g}$ each) were incubated with $200 \mathrm{ng}$ of active NEK2A in kinase buffer $(25 \mathrm{mM}$ HEPES, $\mathrm{pH}$ 7.2, $1 \mathrm{mM}$ DTT, $50 \mathrm{mM} \mathrm{NaCl}, 2 \mathrm{mM}$ EGTA, $5 \mathrm{mM} \mathrm{MgSO}_{4}$ ) with $50 \mu \mathrm{M}$ ATP and $0.5 \mu \mathrm{Ci}$ of $\left[{ }^{32} \mathrm{P}\right]$-ATP. The reaction mixtures $(50 \mu \mathrm{l})$ were incubated at $30{ }^{\circ} \mathrm{C}$ for $30 \mathrm{~min}$ and terminated by adding SDS-PAGE sample buffer. Proteins were then fractionated on SDS-PAGE. The gel was stained with Coomassie Brilliant Blue and quantified by a PhosphoImager (Amersham Biosciences) as previously described [14].

\section{Far-western blotting analysis and pull-down assay}

The far-western blotting assay was carried out as described previously [13]. In brief, aliquots $(\sim 0.5 \mu \mathrm{g})$ of recombinant kinetochore proteins such as MAD1, MAD2, BUB1, BUB3, CENP-E and HsSgol were dipped onto nitrocellulose membrane (Amersham Biosciences). The membrane was blocked with $2 \%$ BSA in PBS containing Tween20 and incubated with $\sim 1 \mu \mathrm{g} / \mathrm{ml}$ of His-tagged NEK2A. After $2 \mathrm{~h}$ incubation, the membrane was washed four times with PBS and incubated with NEK2A antibody for $1 \mathrm{~h}$. Alkaline phosphatase-conjugated secondary antibody was used to detect the primary antibody, and the blot was developed using NBT and BCIP as substrates.

The NEK2A affinity pull-down was previously described [13]. In brief, His-tagged NEK2A immobilized on the Ni-NTA agarose beads (Qiagen) was used as an affinity matrix to incubate with purified GST-HsSgo1. The agarose beads were washed with PBS five times before being boiled in SDS-PAGE sample buffer. The proteins were then fractionated on SDS-PAGE and the gel was stained with Coomassie brilliant blue.

\section{Cell culture and transfection}

HeLa and 293T cells, from American Type Culture Collection (Rockville, MD, USA), were maintained as subconfluent monolayers in DMEM growth medium (Invitrogen, Carlsbad, CA, USA) supplemented with 10\% FBS (Hyclone, Logan, UT, USA) and 100 $\mathrm{U} / \mathrm{ml}$ penicillin plus $100 \mu \mathrm{g} / \mathrm{ml}$ streptomycin (Invitrogen) at $37^{\circ} \mathrm{C}$ with $10 \% \mathrm{CO}_{2}$. Transfections were performed using Lipofectamine 2000 (Invitrogen) exactly as described [13].

\section{Chromosome squashes}

Chromosome squashes were prepared essentially as previously described [15]. In brief, GFP-HsSgol-transfected HeLa cells were treated with $10 \mathrm{ng} / \mathrm{ml}$ nocodazole (Sigma) for $18 \mathrm{~h}$. After arrest, mitotic HeLa cells were harvested by mitotic shake-off and washed with ice-cold PBS. Mitotic HeLa cells were hypotonically swollen for $5 \mathrm{~min}$ at room temperature in $10 \mathrm{vol}$ of PEM buffer containing 5 mM Pipes, pH 7.2, 0.5 mM EDTA, $5 \mathrm{mM} \mathrm{MgCl}_{2}, 5 \mathrm{mM} \mathrm{NaCl}$, and a protease inhibitor cocktail (1 mM PMSF, $2 \mu \mathrm{g} / \mathrm{ml}$ aprotinin, $2 \mu \mathrm{g} / \mathrm{ml}$ pepstatin $\mathrm{A}$, and $2 \mu \mathrm{g} / \mathrm{ml}$ leupeptin) before squashed onto coverslips using Cytospin 4 cytocentrifuge (1 $000 \mathrm{rpm}$ for $2 \mathrm{~min}$; Thermo Scientific). The chromosomes on the coverslips were then fixed with $1 \%$ formaldehyde followed by three washes with PBS. The squashed chromosomes were then stained with anti-centromere antibody (ACA) and countered stained with DAPI (Sigma).

\section{Immunoprecipitation}

To test whether HsSgol forms a complex with NEK2A in vivo, 293 cells were transfected with FLAG-HsSgol and GFP-NEK2A plasmid. Twenty-four hours after the transfection, cells were collected 
and solubilized in lysis buffer (50 mM HEPES, pH 7.4, $150 \mathrm{mM}$ $\mathrm{NaCl}, 2$ mM EGTA, 0.1\% Triton X-100, 1 mM phenylmethylsulfonyl fluoride, $10 \mathrm{~g} / \mathrm{ml}$ leupeptin, and $10 \mathrm{~g} / \mathrm{ml}$ pepstatin A). Lysates were cleared by centrifugation at $16000 \times g$ for $10 \mathrm{~min}$ at $4{ }^{\circ} \mathrm{C}$. FLAGtagged fusion proteins were precipitated by incubating with antiFLAG antibody coupled agarose beads (Sigma). Beads were washed five times with lysis buffer and then boiled in protein sample buffer for $2 \mathrm{~min}$. After SDS-PAGE, proteins were transferred to nitrocellulose membrane. The membrane was divided into three strips and probed with antibodies against the FLAG tag, GFP tag and tubulin, respectively. Immunoreactive signals were detected with ECL kit (Pierce) and visualized by autoradiography on Kodak BioMax film.

\section{Western blotting}

Samples were subjected to SDS-PAGE and transferred onto nitrocellulose membrane. Proteins were probed by appropriate primary and secondary antibodies at concentrations recommended by the manufacturer. Proteins were detected using ECL (Pierce). The band intensity was then scanned using a PhosphorImager (Amersham Biosciences).

To determine whether NEK2A-mediated phosphorylation of HsSgol contributes to its mobility shift in SDS-PAGE, GFP-HsSgol transfected cells or NEK2A siRNA-treated cells were synchronized with $10 \mathrm{ng} / \mathrm{ml}$ nocodazole $24 \mathrm{~h}$ post-transfection. At the end of synchronization, cells were collected and solubilized in SDS-PAGE sample buffer. Samples were subjected to SDS-PAGE and transferred onto nitrocellulose membrane for western blotting analysis with an anti-HsSgol antibody.

\section{Immunofluorescence microscopy}

For immunofluorescence analysis, cells were seeded onto sterile, acid-treated $12 \mathrm{~mm}$ coverslips in 24-well plates (Corning Glass). Twenty-four hours after transfection with various GFP-tagged HsSgo1 plasmids, HeLa cells were pre-extracted with PHEM (100 mM PIPES, $20 \mathrm{mM}$ HEPES, $\mathrm{pH}$ 6.9, $5 \mathrm{mM}$ EGTA, $2 \mathrm{mM} \mathrm{MgCl}_{2}$, and $4 \mathrm{M}$ glycerol) plus $0.2 \%$ Triton $\mathrm{X}-100$ followed by fixation and blocking as described previously $[4,15]$. Cells were then incubated with primary antibody against centromere (ACA) followed by a rhodamine-conjugated secondary antibody. DNA was stained with DAPI (Sigma). Fluorescence labeling was examined under a laser scanning confocal microscope LSM510 (Carl Zeiss) and images were collected and analyzed with Image-5 (Carl Zeiss, Germany).

\section{Results}

\section{HSSgo1 is a novel NEK2A-binding partner}

Our previous study demonstrated that NEK2A is also located to the kinetochore during chromosome congression in mitosis [13]. To delineate the molecular mechanism underlying NEK2A regulation in kinetochore protein-protein interaction networks, we immobilized bacterially expressed recombinant kinetochore proteins onto a nitrocellulous membrane to conduct a quick search for NEK2A-binding proteins using a "high-content" far-western assay [13]. The NEK2A-binding activity was then detected by an anti-NEK2A monoclonal antibody. One advantage of such a "high-content" assay is to screen a large number of
A
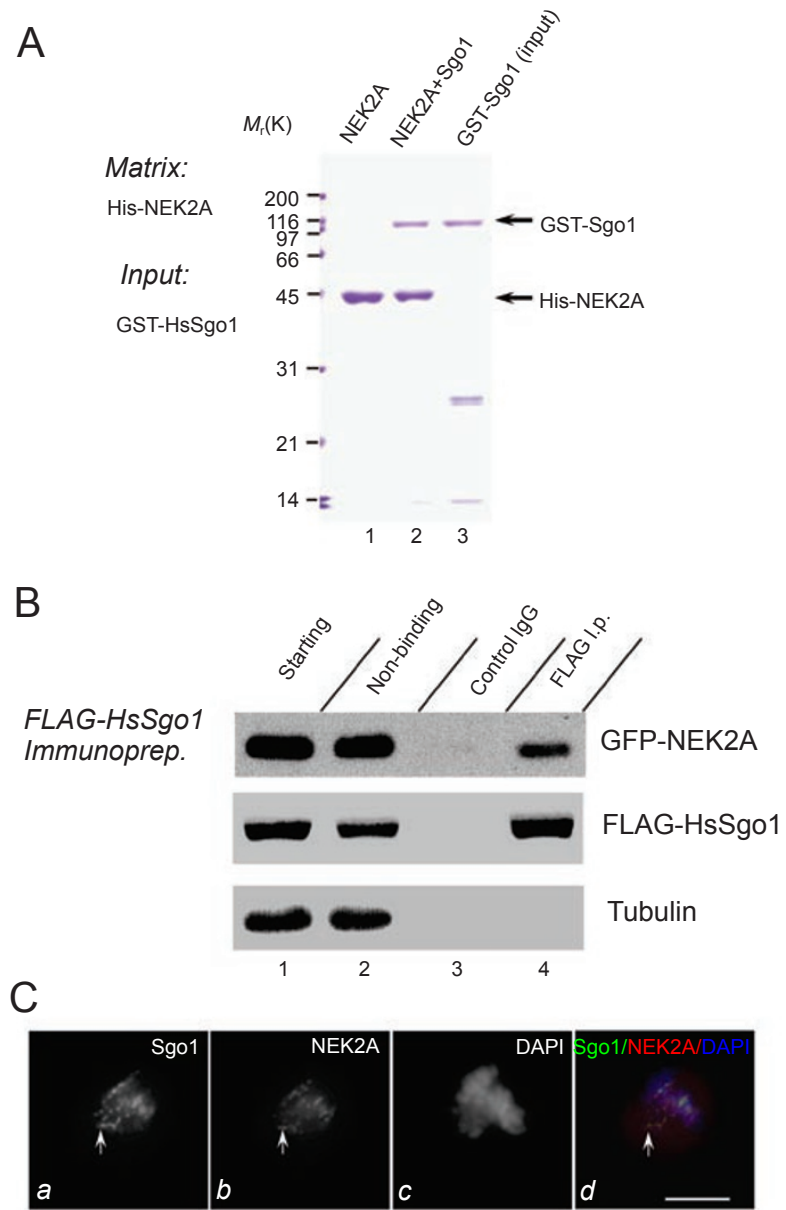

Figure 1 NEK2A interacts with HsSgol and co-distributes to the kinetochore. (A) Reconstitution of NEK2A-HsSgol interaction using recombinant fusion proteins. Histidine-tagged NEK2A recombinant protein purified on Ni NTA-agarose beads was used as affinity matrix for absorbing GST-tagged HsSgol protein purified from bacteria (lane 2). Aliquots of histidine-tagged NEK2A and GST-HsSgol were used as loading controls (lanes 1 and 3). (B) Co-immunoprecipitation of HsSgol and NEK2A from transfected 293T cells. 293T cells co-transfected with a FLAG-tagged hSgol and GFP-tagged NEK2A were extracted and subjected to immunoprecipitation by using monoclonal antibodies to FLAG, respectively. Control immunoprecipitation was performed using a non-specific mouse IgG. Starting fractions, non-binding fractions, and immunoprecipitates were analyzed by SDS-PAGE and immunoblotting using anti-GFP antibody, anti-FLAG antibody and anti-tubulin antibody respectively. Western blotting verifies co-immunoprecipitation of GFP-NEK2A and FLAG-HsSgo1. No tubulin was detected in the FLAG immunoprecipitates. (C) Co-distribution of HsSgol and NEK2A to the kinetochore of mitotic HeLa cells. $a$ - $d$ represent optical images collected from one HeLa cell triply stained with mouse anti-HsSgol antibody (Sgo1, green), DAPI (DNA, blue), rabbit NEK2A antibody (NEK2A, red), and their merged images. Sgol antibody stained centromeres of mitotic HeLa cells as pairs of unresolved double dots ( $a$; arrow). NEK2A staining appears as pairs of clearly resolved double dots ( $b$; arrow). Merge shows a superimposition of NEK2A to that of Sgol staining. Bar, $10 \mu \mathrm{m}$. 
A

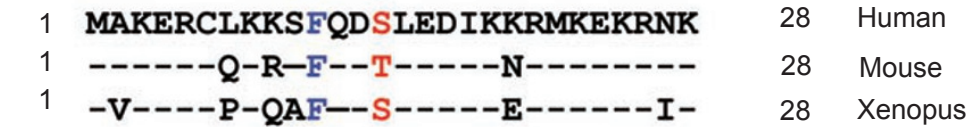

491

SKLRRGDPFTDLCFLNSPIFKQKKDLRRSKKSMKQIQ

527 Human

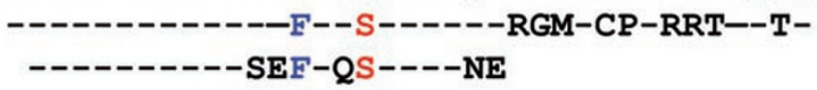

517 Mouse

640 Xenopus

B
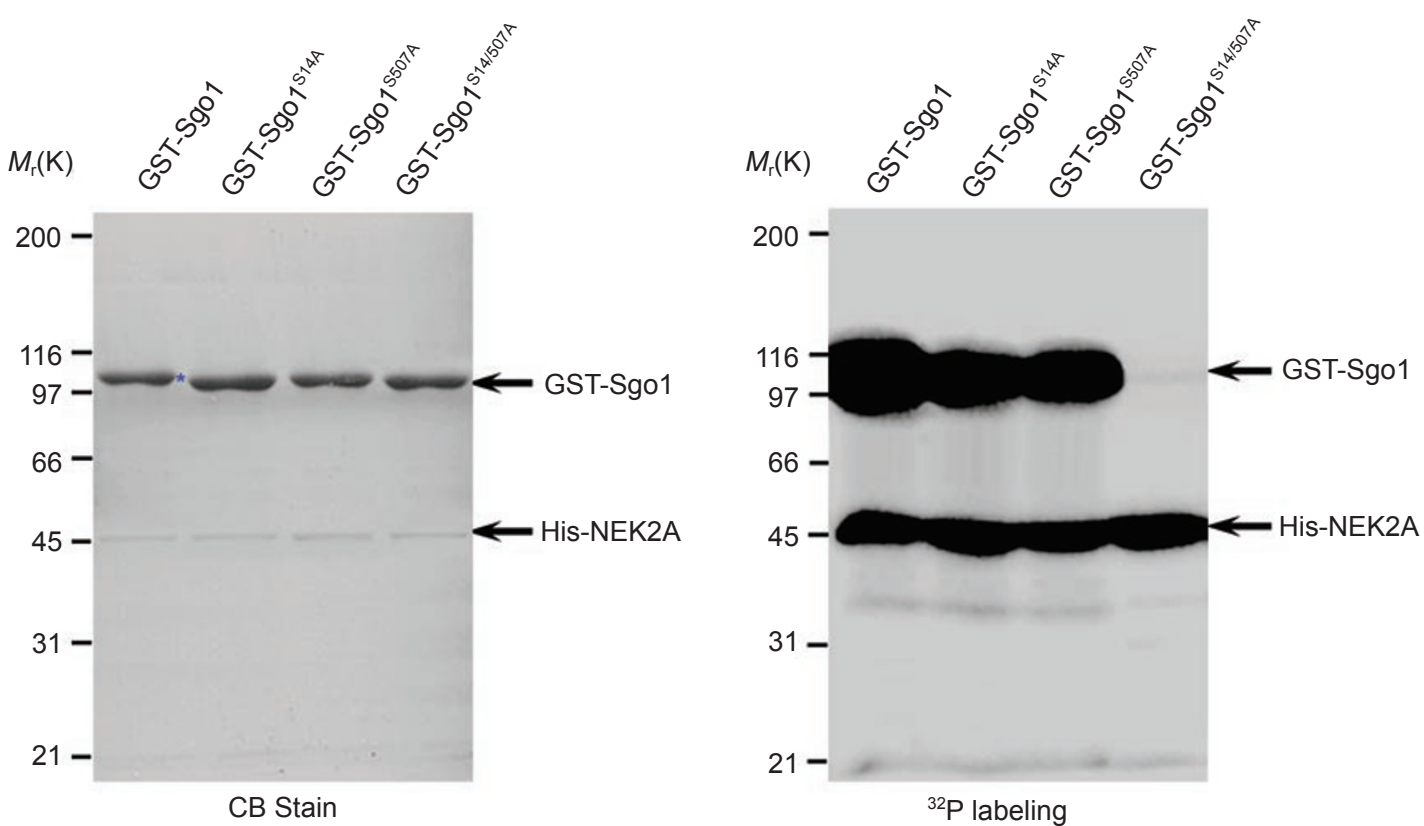

Figure $2 \mathrm{Ser}^{14}$ and $\mathrm{Ser}^{507}$ of HsSgol are substrates of NEK2A. (A) Alignment of conserved NEK2A phosphorylation sites on Xenopus, mouse, and human Sgol proteins. Computational analyses were performed using GPS as described in "Materials and Methods". Based on the GPS prediction, $\operatorname{Ser}^{14}$ and $\mathrm{Ser}^{507}$ are potential NEK2A-mediated phosphorylation sites of HsSgo1. (B) Ser ${ }^{14}$ and Ser ${ }^{507}$ of HsSgol are substrates of NEK2A. Bacterially expressed GST-HsSgol fusion proteins, both wild type and mutants (S14A, S507A and S14/507A), were purified and phosphorylated in vitro using $\left[{ }^{32} \mathrm{P}\right]$-ATP and active NEK2A as described under "Materials and Methods." Samples were separated by SDS-PAGE. Coomassie brilliant blue-stained gel of samples of wild-type GST-Sgo1 plus NEK2A (GST-Sgo1), single mutant S14A GST-Sgo1 plus NEK2A (GST-Sgo1 ${ }^{\text {S14A }}$ ), single mutant S507A GST-Sgo1 plus NEK2A $\left(\right.$ GST-Sgo $\left.1^{\mathrm{S} 507 \mathrm{~A}}\right)$, and double mutant S14/507A GST-Sgo1 plus NEK2A (GST-Sgo1 $\left.{ }^{\mathrm{S} 14 / 507 \mathrm{~A}}\right)$. Note that roughly equivalent amounts of GST-HsSgol proteins were present in the four reactions (left panel). The same gel was dried and subsequently incubated with X-ray film. Note that there was dramatic incorporation of ${ }^{32} \mathrm{P}$ into wild type and single mutant, but not double mutant, HsSgo1 proteins (right panel). In addition, wild-type GST-Sgol protein (marked with blue asterisk) displayed a slower mobility compared to those of non-phosphorylatable mutants, suggesting the mobility shift is a reflection of Sgol protein phosphorylation.

potential interacting proteins in parallel yet avoiding initial intensive labor spent on purifying large quantities of proteins required for pull-down assays. This assay has detected the binding activity of several known NEK2A-interacting proteins, such as Hec1 and Mad1, validating the sensitivity of such an assay (Supplementary Figure 1). This assay is very specific as NEK2A was never found to be associated with GST tag, MBP tag, BSA, or kinetochore protein CENP-E. Interestingly, NEK2A was found to associate with
HsSgol immobilized onto the membrane (Supplementary Figure 1; B8), suggesting a potential interaction between NEK2A and HsSgol.

To validate whether HsSgo 1 binds to NEK2A in solution, we carried out a pull-down assay in which histidine-tagged recombinant NEK2A was purified on Ni-NTA agarose beads and used as an affinity matrix to absorb purified GST-HsSgo1 in test tubes. As shown in Figure 1A, histidine-tagged NEK2A pulled down GST-HsSgo1, suggesting 
a physical interaction between NEK2A and $\mathrm{HsSgol}$ in vitro. To examine whether HsSgol forms a complex with NEK2A in cells, we carried out an immunoprecipitation assay using mitotic lysates from $293 \mathrm{~T}$ cells transiently transfected to express FLAG-HsSgol and GFP-NEK2A. As shown in Figure 1B, western blot using GFP antibody confirmed that GFP-NEK2A is pulled down by FLAG-HsSgol (upper panel; lane 4) while FLAG antibody blotting confirmed a successful precipitation of HsSgol (middle panel; lane 4). GFP-tagged NEK2A was not precipitated with control IgG (lane 3), nor was tubulin detected in any of the immunoprecipitates. Thus, we conclude that HsSgol interacts with NEK2A in vitro and in vivo.

If $\mathrm{HsSgo} 1$ is a cognate binding partner of NEK2A, they should co-distribute to the kinetochore of prometaphase cells. To test this, we performed an immunocytochemical staining in which an anti-HsSgol mouse antibody and an anti-NEK2A rabbit antibody were employed to mark their kinetochore distribution. As shown in Figure 1C, HsSgol and NEK2A displayed a typical kinetochore distribution (arrows $\mathrm{a}$ and $\mathrm{b}$ ) and the merged image confirmed their codistribution to the kinetochore of mitotic cells (arrow $d$ ).

\section{HsSgol is a novel substrate of NEK2A}

Our previous studies show that mitotic kinase NEK2A functions as a novel integrator of the spindle checkpoint signaling [13]. Since HsSgol binds to NEK2A in vitro and in vivo, we sought to test whether HsSgol is a substrate of NEK2A. Our computational analysis suggests that $\mathrm{Ser}^{14}$ and $\mathrm{Ser}^{507}$, which are conserved among vertebrate HsSgol proteins, are potential substrates of NEK2A [16] (Figure 2A). Our mass spectrometric analysis of HsSgol immunoprecipitates also pointed to the possibility of $\mathrm{Ser}^{14}$ and $\mathrm{Ser}^{507}$ phosphorylation in nocodazole-synchronized HeLa cells. To test whether $\mathrm{Ser}^{14}$ and $\mathrm{Ser}^{507}$ are substrates of NEK2A, we performed an in vitro phosphorylation assay on recombinant GST-HsSgo1 fusion proteins, including both wild-type protein and non-phosphorylatable mutants in which $\mathrm{Ser}^{14}$ and $\mathrm{Ser}^{507}$ were either individually or both replaced by alanine $\left(\mathrm{HsSgo1}^{\mathrm{S14A}}, \mathrm{HsSgo1}^{\mathrm{S} 507 \mathrm{~A}}\right.$, and $\left.\mathrm{HsSgo1}^{\mathrm{S1} 1 / 507 \mathrm{~A}}\right)$. Both GST fusion proteins, wild type and mutant $\mathrm{HsSgo}{ }^{\mathrm{S} 14 / 507 \mathrm{~A}}$, migrate at about the predicted 105 $\mathrm{kDa}$ as shown in Figure 2B (left panel). Incubation of the fusion proteins with $\left[{ }^{32} \mathrm{P}\right]$-ATP and His-NEK2A resulted in the incorporation of ${ }^{32} \mathrm{P}$ into wild type, $\mathrm{HsSgol}^{\mathrm{S} 14 \mathrm{~A}}$ and $\mathrm{HsSgo}^{\mathrm{S} 507 \mathrm{~A}}$ proteins but not $\mathrm{HsSgo}{ }^{\mathrm{S14/507 \textrm {A }}}$ mutant (Figure $2 \mathrm{~B}$, right panel). This NEK2A-mediated phosphorylation is specific, since incubation of $\mathrm{HsSgo} 1$ with $\left[{ }^{32} \mathrm{P}\right]$-ATP in the absence of NEK2A resulted in no detectable incorporation of radioactivity into the wild-type protein (data not shown). Thus, we conclude that both $\mathrm{Ser}^{14}$ and $\mathrm{Ser}^{507}$ on HsSgol are substrates of NEK2A in vitro.
Localization of HsSgol to the kinetochore does not require NEK2A

Interestingly, wild-type HsSgol treated with NEK2A displayed a slower mobility compared to that of non-phosphorylatable $\mathrm{HsSgo1}{ }^{\mathrm{S} 14 / 507 \mathrm{~A}}$ mutant (Figure 2B; compare lanes 1 with 4), suggesting a possible phosphorylation-mediated mobility shift induced by NEK2A. To test whether endogenous HsSgol mobility is a function of NEK2A, we carried out western blot of HsSgol from HeLa cells treated with NEK2A siRNA followed by nocodazole synchronization. As shown in Figure 3A, the mobility shift of HsSgol was confirmed to be NEK2A-dependent as depletion of NEK2A retained HsSgol to the faster migrating form, indicating that endogenous HsSgol is phosphorylated by NEK2A in mitosis.

To examine whether phosphorylation of $\mathrm{Ser}^{14}$ and $\mathrm{Ser}^{507}$ is responsible for the mobility shift of HsSgol in mitosis, we carried out western blot analysis of HsSgol using HeLa cells transiently transfected to express GFP-tagged wild-type HsSgol and mutant $\mathrm{HsSgo} 1 \mathrm{HsSgo}^{\mathrm{S} 14 / 507 \mathrm{~A}}$ and $\left.\mathrm{HsSgo1}^{\text {S14/507D }}\right)$. As expected, wild-type GFP-HsSgol protein migrated more slowly compared to mutant proteins GFP-HsSgo $1^{\text {S14/507A }}$ and HsSgo $1^{\text {S14/507D }}$ while endogenous $\mathrm{HsSgol}$ proteins from these three transfectants displayed similar mobility, suggesting that the mobility shift is a function of phosphorylation on $\mathrm{Ser}^{14}$ and $\mathrm{Ser}^{507}$ (Figure 3B; lanes 1-3). Thus, we conclude that HsSgol is phosphorylated by NEK2A at $\mathrm{Ser}^{14}$ and $\mathrm{Ser}^{507}$ in mitosis.

To evaluate the efficacy of exogenous HsSgol expression, we carried out western blotting analysis using anti$\mathrm{HsSgol}$ antibody to compare the levels of exogenously expressed GFP-HsSgol proteins to that of endogenous HsSgo1. As shown in Figure 3B, exogenously expressed GFP-HsSgol proteins were about twice the level of endogenous HsSgol proteins in these transfected HeLa cells. Given a typical $\sim 50 \%$ transfection efficiency, the actual expression level of GFP-HsSgol in positively transfected cells is about four-fold higher than that of endogenous protein [17].

To examine whether NEK2A-mediated phosphorylation is essential for HsSgol localization to the kinetochore, plasmids expressing GFP-tagged wild type, non-phosphorylatable, and phospho-mimicking HsSgol were transfected into HeLa cells, and fusion proteins were scored for their kinetochore localization relative to the centromere marker ACA under fluorescence microscopy. The transfected cells were triply stained or analyzed for ACA (red), GFPHsSgol (green) and DNA (blue). As shown in Figure 3C, the kinetochore distribution of exogenously expressed GFP-HsSgol proteins (wild type, HsSgo $1^{\mathrm{S} 14 / 507 \mathrm{~A}}$, and $\mathrm{HsSgol}^{\mathrm{S14/507D}}$ ) was readily observed as the merged images show the co-localization of GFP-HsSgol proteins with the 
A

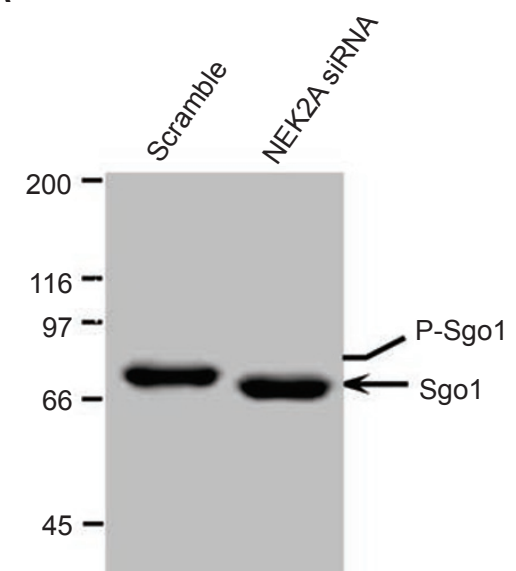

Sgo1 blot

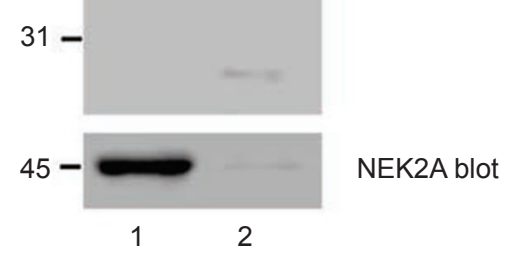

C
B
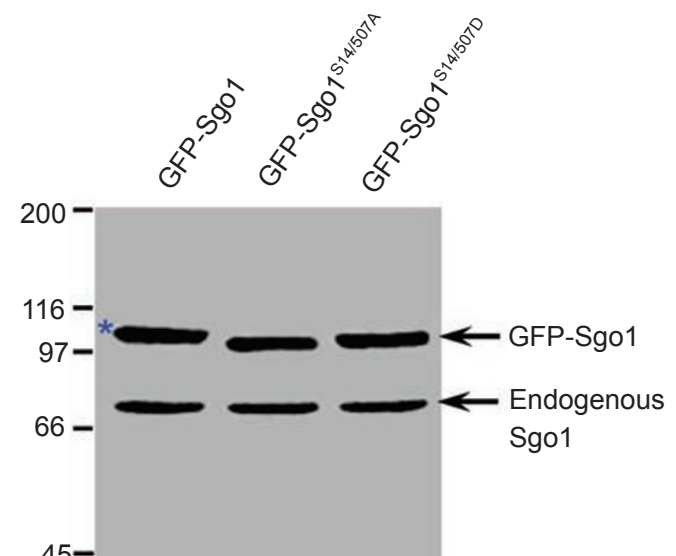

45-

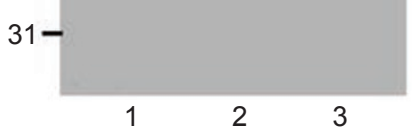

Sgo1 blot

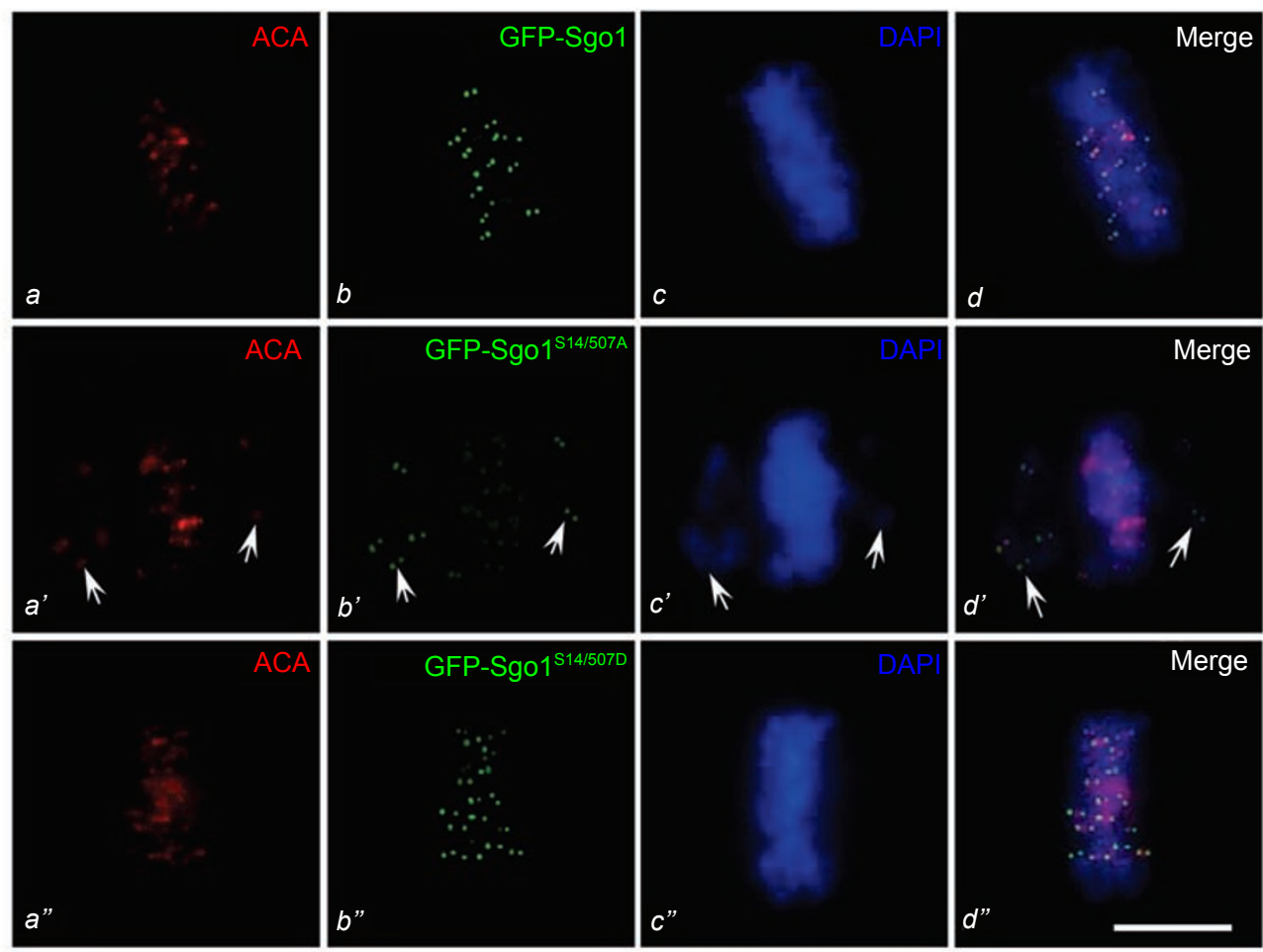


D

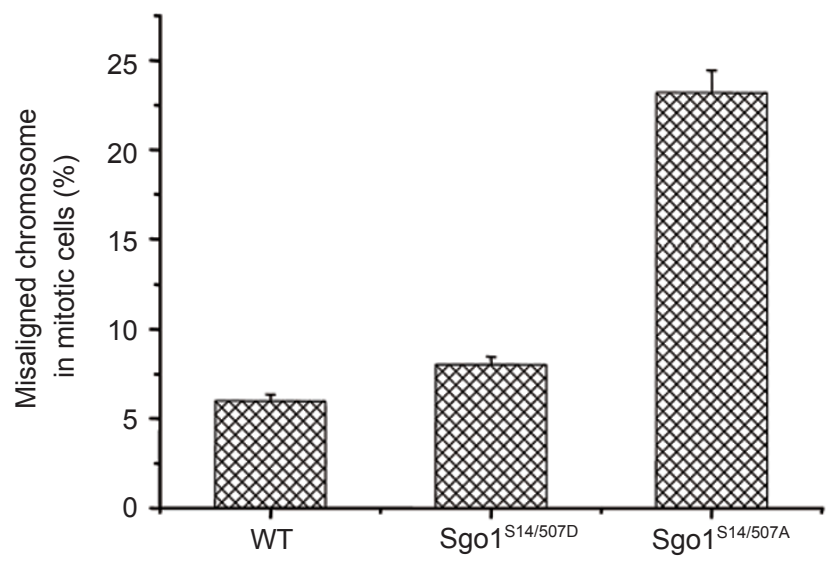

E
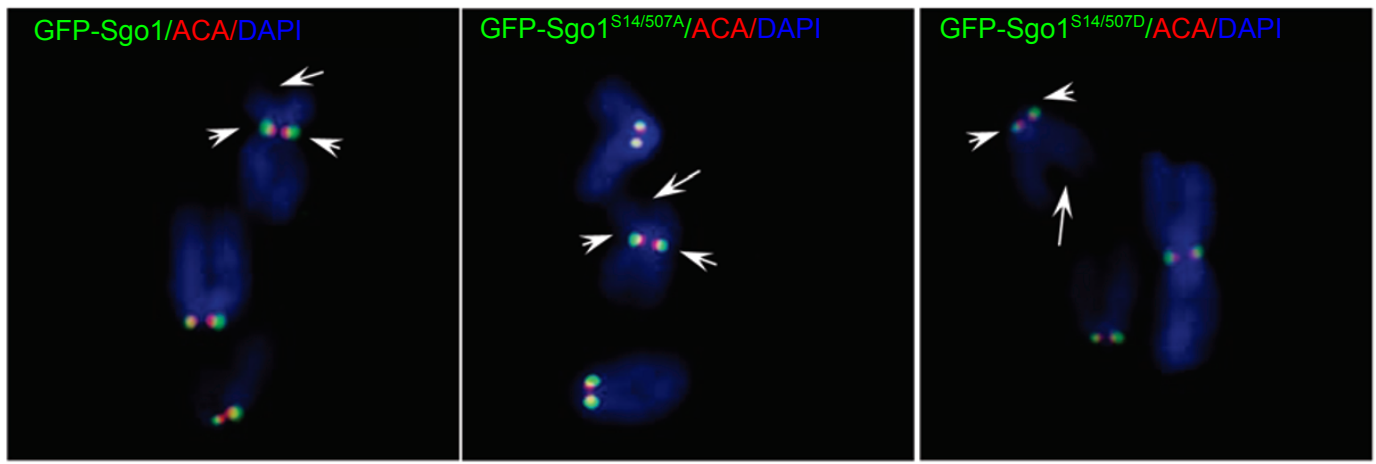

Figure 3 NEK2A-mediated phosphorylation of HsSgol is essential for faithful chromosome congression. (A) HsSgol is a substrate of NEK2A in mitosis. Aliquots of HeLa cells were transfected with NEK2A siRNA and scramble control as described under "Materials and Methods". Twenty-four hours after the transfection, these cells were synchronized with $10 \mathrm{ng} / \mathrm{ml}$ nocodazole for additional $18 \mathrm{~h}$. Cells were then harvested for SDS-PAGE and subsequent western blotting analyses of Sgol (upper panel) and efficiency of NEK2A depletion (lower panel). Note that Sgol protein in scramble-transfected cells displayed a slower mobility compared to that of the NEK2A-depleted sample, suggesting that the mobility shift is an indicator of NEK2A-mediated phosphorylation of Sgo1. (B) Exogenously expressed GFP-Sgo1 protein behaves like endogenous Sgo1. Samples from both wild type and mutant Sgo1-transfected HeLa cells were prepared and separated on SDS-PAGE, blotted to nitrocellulose, and probed by an anti-HsSgo1 antibody. The Sgo1 antibody recognizes both endogenous $(75 \mathrm{kDa})$ and exogenously expressed GFP-Sgol (105 kDa) proteins. Note that wild-type GFP-Sgol protein displayed a slower mobility compared to those of mutants, suggesting exogenously expressed GFP-Sgol protein behaves like endogenous Sgo1. (C) Overexpression of non-phosphorylatable HsSgol resulted in chromosome congression defects. This triple montage and their merges represent mitotic HeLa cells transiently transfected to express wild type and phospho-mutant HsSgol proteins. The transfected cells were pre-extracted before fixation as described under "Materials and Methods" and stained for ACA (red), GFP-Sgol (green), and DAPI (blue). Note that chromosomes failed to congress and align at the spindle equator in non-phosphorylatable Sgo1-expressing cells (arrows; $a^{\prime}-d^{\prime}$ ) and misaligned chromosome is correlated with higher expression of GFP-Sgo $1^{\text {S14/507A }}$ compared to those near the spindle equator. Bar, $10 \mu \mathrm{m}$. (D) Phosphorylation of the HsSgo1 by NEK2A is required for faithful chromosome movement. HeLa cells were transfected with GFP-tag wild-type hSgo1, Sgo ${ }^{\text {S14/507A }}$, and Sgo1 ${ }^{\text {S14/507D }}$ for 48 $\mathrm{h}$ followed by fixation and DNA staining. Cells were then examined under fluorescence microscopy to determine the phenotypes. Cells in mitosis and those that carried misaligned chromosomes were quantified and expressed as the percentage of total mitotic cells. An average of 150 mitotic cells from three separate experiments were counted. Error bars represent S.E.; $n=3$ preparations. $p<0.001$. (E) Centromeric cohesion is independent of NEK2A-mediated phosphorylation of HsSgo1. Chromosome squashes from nocodazole-treated HeLa cells transiently transfected to express wild type, non-phosphorylatable and phospho-mimicking HsSgol proteins were prepared as described under "Materials and Methods". The chromosomes were fixed and stained to mark centromeres and DNA. Despite the separation of chromosome arms seen in all Sgo1-expressing cells, centromeric conhesion remained intact. 
centromere marker ACA (Figure 3C, $d, d^{\prime}$ and $d$ '), suggesting that exogenous $\mathrm{HsSgol}$ proteins are able to target to the kinetochore regardless of the phosphorylation status of $\operatorname{Ser}^{14}$ and $\mathrm{Ser}^{507}$. These data suggest that phosphorylation of $\mathrm{Ser}^{14}$ and $\mathrm{Ser}^{507}$ by NEK2A is not required for targeting of $\mathrm{HsSgol}$ to the kinetochore.

\section{NEK2A-mediated phosphorylation of HSSgol is required for faithful chromosome congression}

Interestingly, at comparable time points, many nonphosphorylatable HsSgo $1^{\text {S14/507A }}$-expressing cells contained misaligned chromosomes that were readily observed (Figure $3 \mathrm{C}, a^{\prime}-d^{\prime}$; arrows). Examination of $\sim 100$ mitotic cells expressing $\mathrm{HsSgo}^{\mathrm{S14/507 \textrm {A }}}$ revealed that many chromosomes in almost all positively transfected cells were positioned close to spindle poles, with some near the spindle equator (Figure 3C, $c^{\prime}$ ). This pattern is the expected distribution for chromosomes that are actively congressing [4]. In fact, misaligned chromosomes bear double-dot staining of HsSgol (Figure 3C, $b^{\prime}$ ), suggesting that these chromosomes near the poles were not separated. We surveyed approximately 150 mitotic cells, in which both sets of sister chromatids were in the same focal plane, from cells expressing the three GFP-HsSgol proteins (wild type, S14/507A, and S14/507D mutant). As shown in Figure 3D, expression of the non-phosphorylatable Sgo1 resulted in a significant increase in cells bearing misaligned chromosomes (23.2 $\pm 6.6 \% ; p<0.05)$. Only a minority of GFP-HsSgol- and GFP-Sgo ${ }^{\text {S14/507D }}$-expressing cells displayed misaligned chromosomes $(6 \pm 1.9 \%$ for GFP-HsSgol and $8 \pm 2.5 \%$ for GFP-Sgo $1^{\text {S14/507D }}$ ). Thus, we conclude that phosphorylation of HsSgo 1 by NEK2A is essential for faithful chromosome congression.

In vertebrates, sister chromatid cohesion is orchestrated by the associations at the centromere and chromosome arms. Depletion of HsSgol in HeLa cells causes massive mis-segregation of sister chromatids due to an aberrant centromeric cohesion [18]. To evaluate the functional role of NEK2A-mediated phosphorylation in centromeric cohesion, we prepared chromosome spreads of HeLa cells from GFP-HsSgo1-expressing cells synchronized by nocodazole treatment and analyzed the morphology of chromosomes whose centromeres were marked with GFPHsSgol proteins. To facilitate our analysis, chromosomes were counterstained with ACA to confirm the centromeric cohesion. As shown in Figure 3E, most sister chromatids in GFP-HsSgo 1 expressing cells remained attached at their centromeres while chromosome arms are separated, consistent with the fact that most of the cohesin residing along the chromosome arms is removed in prophase (e.g. Ref [19]). Thus, we concluded that phosphorylation of Sgo1 by NEK2A is not involved in centromeric cohesion.
NEK2A phosphorylation of HsSgol is essential for a proper chromosome-microtubule attachment

Sgo1 is a microtubule binding protein [6], and our observations of delayed chromosome congression in GFP-HsSgo $1^{\text {S14/507A }}$-overexpressing cells suggest that NEK2A-mediated phosphorylation is required for a stable association between the kinetochores and spindle microtubules. To validate this hypothesis, we probed for the presence of cold-stable kinetochore microtubules in GFP-HsSgo $1^{\text {S14/507A }}$-overexpressing cells. In brief, treatment of mitotic cells on ice during permeabilization prior to fixation destabilizes non-kinetochore microtubules, leaving behind mainly kinetochore fibers (e.g. Ref [4]). As shown in Figure 4A, the spindle remained intact after cold treatment, with microtubule fibers clearly attached to each kinetochore stained with ACA (Figure 4A, $a$, red), in cells overexpressing wild-type HsSgol. In $\mathrm{HsSgol}^{\text {S14/507A }}$ overexpressing cells, cold-stable kinetochore-microtubule fibers were present on both aligned chromosomes and chromosomes near the pole (Figure 4A, $b$ ', red). Quantitative analysis of fluorescence intensity of GFP-HsSgo $1^{\mathrm{S} 14 / 507 \mathrm{~A}}$ indicates that the fluorescence intensity of the kinetochore near the pole is about 5.3-fold higher than that of an aligned kinetochore, consistent with the notion that overexpression of $\mathrm{HsSgo} 1^{\mathrm{S} 14 / 507 \mathrm{~A}}$ produces a "dominant"-negative effect in chromosome alignment.

We next examined the microtubule attachment and the orientation of sister kinetochores on chromosomes in the cells overexpressing GFP-HsSgo $1^{\mathrm{S} 14 / 507 \mathrm{~A}}$ mutant. To this end, we performed confocal scanning microscopy on HeLa cells fixed and stained with anti-tubulin antibody and ACA. To show individual kinetochores more clearly, we present optical sections in insets while using maximal intensity projections of the entire Z-stack for volume views. We scored kinetochore attachment in which both kinetochores were in the same focal plane, in both wild-type GFP-HsSgol and GFP-HsSgo $1^{\text {S14/507A }}$-expressing cells. As shown in Figure 4A, it is readily apparent from the confocal projection that overexpression of the GFP-HsSgo $1^{\mathrm{S} 14 / 507 \mathrm{~A}}$ mutant resulted in misaligned chromosome near the poles while all chromosomes were aligned around the equator in the cells expressing wild-type HsSgol. Enlargement of kinetochore-microtubule attachment indicates that some cold-stable microtubules display syntelic orientation and monotelic orientation (illustrated in Figure 4B). In contrast, most spindle microtubules exhibit amphitelic configuration in control cells expressing wild-type HsSgo1. Our measurement indicates no difference of the fluorescence intensity of wild-type GFP-HsSgol between aligned and misaligned kinetochore (data not shown). However, the misaligned chromosome is correlated with the higher expression level of $\mathrm{HsSgo1}^{\mathrm{S14/507 \textrm {A }}}$ protein as judged by the fluorescence 
A
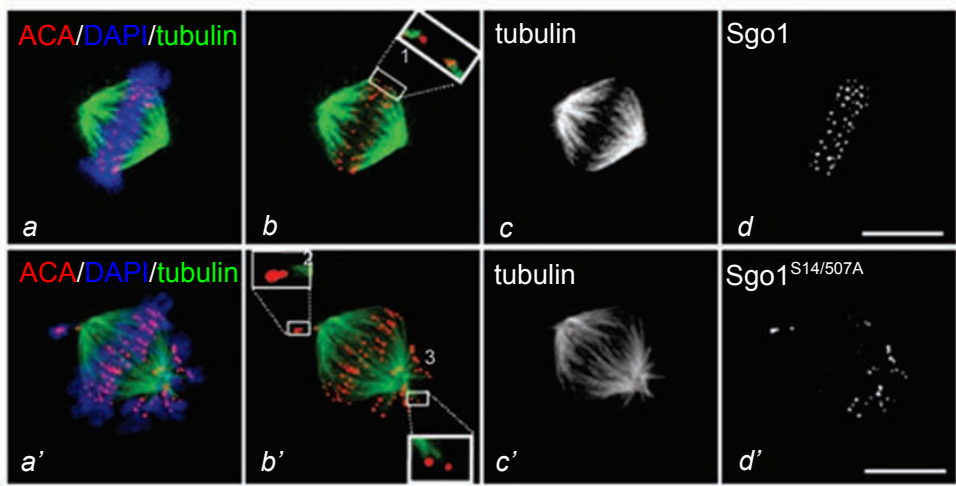

$\mathrm{SgO}^{\mathrm{S1} 14507 \mathrm{~A}}$

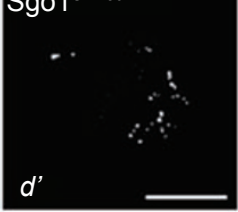

$\mathrm{B}$
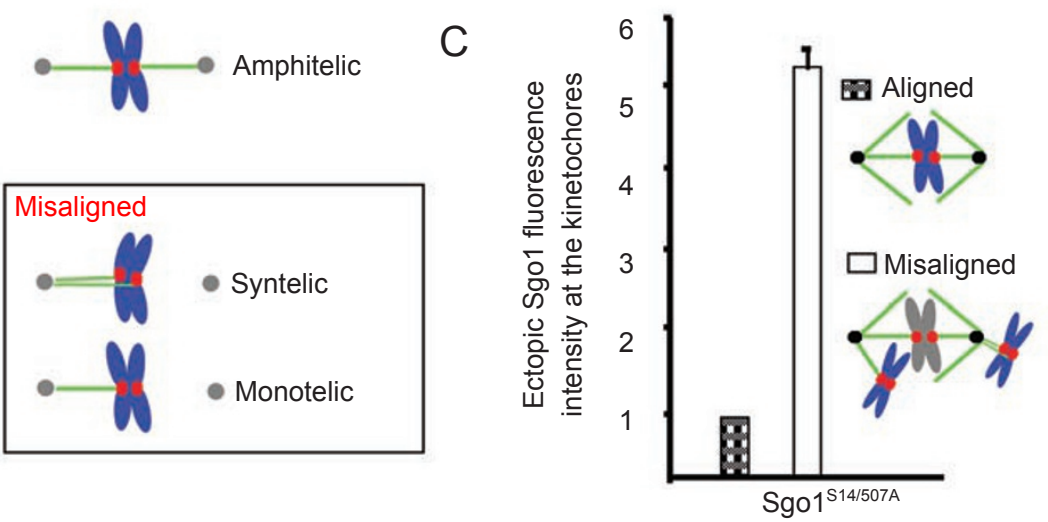

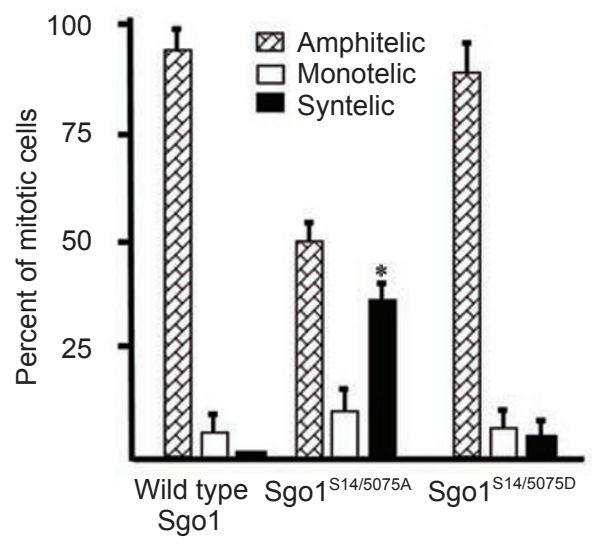

Figure 4 Inhibition of HsSgol phosphorylation results in aberrant kinetochore-microtubule attachments. (A) Indirect immunofluorescence images of HeLa cells expressing wild-type hSgol $(a-d)$ or Sgol ${ }^{\text {S14/507A }}\left(a^{\prime}-d^{\prime}\right)$. Cells were kept on ice for 7 min, permeabilized for $90 \mathrm{~s}$ in PHEM, then fixed and immunostained. Cold treatment selectively removes non-kinetochore microtubules. Each chromosome (blue) has two sister kinetochores (red) that are stained with the ACA. In HeLa cells expressing wild-type HsSgo1 $(a-d)$, almost every pair of sister kinetochores are attached by microtubules (green) emanating from the opposing poles. However, in $\mathrm{HsSgo}^{\mathrm{S} 14 / 507 \mathrm{~A}}$-expressing cells $\left(a^{\prime}-d^{\prime}\right)$, several examples with both kinetochores of a sister pair attached to microtubules emanating from the same pole are observed $\left(b^{\prime}\right) .1,2$, and 3 show higher magnification micrographs for three pairs of kinetochores either properly (in 1) or aberrantly (in 2 and 3) attached to spindle microtubules. (B) Schematic illustration of various kinetochore-microtubule attachment orientations seen in mitosis. The boxed orientations are typical misalignment seen in HsSgo $1^{\text {S14/507A }}$-expressing cells. (C) Quantification of Sgo1 ${ }^{\text {S14/507A }}$ fluorescence intensity at kinetochores of misaligned chromosomes compared to that of chromosomes aligned at the equator. Because of the difficulty in distinguishing syntelic orientation from monotelic orientation in some cases, we grouped these two orientations as misalignment. (D) Quantification of kinetochore-microtubule attachment orientation in HeLa cells expressing wild-type Sgo1, Sgo $1^{\mathrm{S} 14 / 507 \mathrm{~A}}$, and $\mathrm{Sgo} 1^{\mathrm{S} 14 / 507 \mathrm{D}}$ respectively. Error bars represent S.E.; $n=3$ preparations $(\sim 100$ mitotic cells per preparation), $p<0.001$. 
intensity (Figure 4C), suggesting that misaligned chromosome is a function of $\mathrm{HsSgol}^{\mathrm{S} 14 / 507 \mathrm{~A}}$ overexpression. We surveyed approximately 100 mitotic cells for kinetochore attachment orientation, in which both kinetochores were in the same focal plane, from $\mathrm{HsSgo1}^{\mathrm{S} 14 / 507 \mathrm{~A}}$-overexpressing cells and the controls. Our survey indicates that syntelic orientation is a typical feature of $\mathrm{HsSgo}^{\mathrm{S} 14 / 507 \mathrm{~A}}$-overexpressing cells. As shown in Figure 4D, a summary from three different experiments shows that expression of nonphosphorylatable HsSgo 1 resulted in significant increases in cells bearing syntelic attachment $(35.3 \pm 4.7 \%)$, compared to the control $(0.8 \pm 0.1 \%$ for wild type). Therefore, we conclude that NEK2A-mediated phosphorylation of $\mathrm{HsSgol}$ at $\mathrm{Ser}^{14}$ and $\mathrm{Ser}^{507}$ is essential for proper attachment of the kinetochore to spindle microtubules.

\section{Discussion}

We have identified that the function of HsSgol is regulated by NEK2A, a kinetochore-associated kinase essential for cell division $[4,13]$. Although NEK2A-mediated phosphorylation of HsSgol is not essential for its localization to the kinetochore, inhibition of $\mathrm{HsSgol}$ phosphorylation resulted in chromosome congression defects and aberrant kinetochore-microtubule attachments. These findings offer a mechanistic link between the NEK2A-mediated phosphorylation of HsSgol and proper attachment of spindle microtubules to kinetochores.

Our recent study has revealed the critical role of NEK2A in chromosome segregation in mammalian cells via its interaction with spindle checkpoint protein MAD1 [13]. To delineate the molecular mechanism underlying NEK2A regulation in kinetochore interaction, we carried a "high-content" screen for NEK2A interacting proteins and identified HsSgol as its cognate substrate. Despite our corroborative studies showing that HsSgol is phosphorylated by NEK2A at $\mathrm{Ser}^{14}$ and $\mathrm{Ser}^{507}$ in vitro and in vivo, it remains to be determined how the spatio-temporal order of this NEK2A-mediated HsSgol phosphorylation is orchestrated as majority of NEK2A kinase activity is minimized in prometaphase [11]. It would be of great interest to develop phosphorylation site-specific antibody to illustrate the phosphorylation and dephosphorylation dynamics of HsSgol in mitosis.

While NEK2A-mediated protein phosphorylation of $\mathrm{HsSgol}$ at $\mathrm{Ser}^{14}$ and $\mathrm{Ser}^{507}$ is not required for the localization of HsSgol at the kinetochore, overexpression of the non-phosphorylatable GFP-HsSgo1 ${ }^{\text {114/507A }}$ mutant results in a significant increase in attachment errors, including syntelic attachment and monotelic attachment. The aberrant attachment is a function of expression of the non-phosphorylatable GFP-HsSgo $1^{\mathrm{S} 14 / 507 \mathrm{~A}}$ mutant as expression of wild-type GFP-HsSgol did not perturb the amphitelic attachment. However, it is unclear whether accumulation of the HsSgo $1^{\mathrm{S1} 1 / 507 \mathrm{~A}}$ mutant protein in the kinetochore caused the chromosome congression defects or $\mathrm{HsSgol}^{\mathrm{S} 14 / 507 \mathrm{~A}}$ mutant protein became accumulated at the misaligned kinetochore as a "sensor" to the aberrant attachment. In any event, it would be of great importance to investigate whether and how overexpression of $\mathrm{HsSgol}^{\mathrm{S14/507 \textrm {A }}} \mathrm{mu}-$ tant causes the mal-orientation. In addition, it would be equally important to ascertain whether the enrichment of HsSgo $1^{\text {S14/507A }}$ mutant protein in misaligned kinetochore is only a reporter for aberrant orientation. This will require real-time imaging of both kinetochore movements and $\mathrm{HsSgo} 1^{\mathrm{S} 14 / 507 \mathrm{~A}}$ molecular dynamics.

In contrast to depletion of $\mathrm{HsSgol}$ using siRNA, expression of the non-phosphorylatable $\mathrm{HsSgo} 1^{\text {S14/507A }}$ mutant did not alter the centromeric cohesion (Figure 3E), and the localization of outer kinetochore proteins such as CENP-E and CENP-F (Fu et al., unpublished observation). It remains to be established whether phosphorylation of HsSgol is required to regulate kinetochore microtubule dynamics in prometaphase and how such a phosphorylation regulates the kinetochore-microtubule attachment stability. Protein phosphorylation is a dynamic process and the status of phosphorylation is orchestrated by kinase and phosphatase. Despite the fact that NEK2A is degraded at prometaphase [11], the NEK2A-mediated phosphorylation of HsSgol remains (Figure 3A) and is essential for chromosome congression to the spindle equator at metaphase (Figure 3C). One possibility is that the NEK2A-induced phospho-epitopes remain on HsSgol despite the degradation of NEK2A, as the phosphatase responsible for dephosphorylation of HsSgol is inactive until chromosomes achieve metaphase alignment. To this end, it would be necessary to develop phospho-epitope antibody specific for $\mathrm{Ser}^{14}$ and $\mathrm{Ser}^{507}$ and illustrate the temporal profile of HsSgol phosphorylation. Our study here aims to provide an outline so that further mechanistic studies of phospho-regulation of HsSgol can be pursued.

Loss or gain of whole chromosomes, the form of chromosomal instability most commonly associated with human cancers, is expected to arise from the failure to accurately segregate chromosomes in mitosis. The mitotic checkpoint is one pathway that prevents segregation errors by blocking the onset of anaphase until all chromosomes make proper attachments to the spindle. Another process that prevents errors is stabilization and destabilization of connections between kinetochore and spindle microtubules, in which spatiotemporal regulation of kinetochore organization and microtubule attachment are integrated into mitotic events. The question remains as to how these two pathways are coordinated to ensure accurate chromosome segregation. 
We observed that many chromosomes in $\mathrm{HsSgol}^{\mathrm{S} 14 / 507 \mathrm{~A}}$ expressing cells show syntelic orientation, with both kinetochores attached to the cold-stable spindle microtubules originating from a single pole. Syntelic orientation could result from the collapse of a bipolar spindle, but our

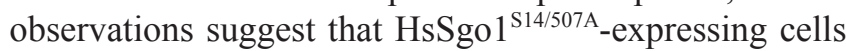
entering mitosis gain this syntelic orientation by de novo capture of microtubules by both kinetochores in a sister pair. Two mechanisms for correction of syntelic error have been proposed from meiotic studies, where the first event is the microtubule capture from the opposite pole [20] or microtubule release at the attached pole [21]. In this regard, it would be of great interest to systematically study phospho-regulation of $\mathrm{HsSgo} 1$ in real-time to examine whether and how syntelic orientation is corrected in mitosis.

Taken together, our finding of phospho-regulation of HsSgo 1 by NEK2A demonstrates a critical role of HsSgo 1 in kinetochore dynamics in addition to its function at kinetochore-microtubule attachment. The fact that suppression of HsSgo 1 phosphorylation abrogates microtubule attachment to the kinetochore and induces chromosome congression defect and mis-segregation in HeLa cells prematurely exiting from anaphase (Fu et al., unpublished observation), demonstrates the importance of HsSgol phosphorylation in faithful chromosome segregation and the maintenance of genomic stability in mitosis.

\section{Acknowledgments}

We thank members of our group for insightful discussion during the course of this study. This work was supported by grants from Chinese Academy of Science (KSCX1YW-R65; KSCX2-YW-H10), National Basic Research Program of China (2002CB713700), Hi-Tech Research and Development Program of China (2001AA215331), Chinese Minister of Education (20020358051 to XY; PCSIRT0413 to XD), National Natural Science Foundation of China (39925018; 30270293 to XY; 30500183 to XD; 30600222 to JY), and National Institutes of Health (USA) (DK56292; CA92080) to XY (a Georgia Cancer Coalition Eminent Scholar). JY was supported by China Postdoctor (2005037560).

\section{References}

1 Cleveland DW, Mao Y, Sullivan KF. Centromeres and kinetochores: from epigenetics to mitotic checkpoint signaling. Cell 2003; 112:407-421.

2 Nicklas RB. The motor for poleward chromosome movement in anaphase is in or near the kinetochore. J Cell Biol 1989; 109:2245-2255.

3 Rieder CL, Alexander SP. Kinetochores are transported poleward along a single astral microtubule during chromosome attachment to the spindle in newt lung cells. J Cell Biol 1990; 110:81-95.
4 Yao X, Abrieu A, Zheng Y, et al. CENP-E forms a link between attachment of spindle microtubules to kinetochores and the mitotic checkpoint. Nat Cell Biol 2000; 2:484-491.

5 Kitajima TS, Kawashima SA, Watanabe Y. The conserved kinetochore protein shugoshin protects centromeric cohesion during meiosis. Nature 2004; 427:510-517.

6 Salic A, Waters JC, Mitchison TJ. Vertebrate shugoshin links sister centromere cohesion and kinetochore microtubule stability in mitosis. Cell 2004; 118:567-578.

7 McGuinness BE, Hirota T, Kudo NR, et al. Shugoshin prevents dissociation of cohesin from centromeres during mitosis in vertebrate cells. PLoS Biol 2005; 3:e86.

8 Katis VL, Galova M, Rabitsch KP, et al. Maintenance of cohesin at centromeres after meiosis I in budding yeast requires a kinetochore-associated protein related to MEI-S332. Curr Biol 2005; 14:560-572.

9 Marston AL, Tham WH, Shah H, et al. A genome-wide screen identifies genes required for centromeric cohesion. Science 2004; 303:1367-1370.

10 Rabitsch KP, Gregan J, Schleiffer A, et al. Two fission yeast homologs of Drosophila Mei-S332 are required for chromosome segregation during meiosis I and II. Curr Biol 2004; 14:287301 .

11 Nigg EA. Mitotic kinases as regulators of cell division and its checkpoints. Nat Rev Mol Cell Biol 2001; 2:21-32.

12 O'Connell MJ, Krien MJ, Hunter T. Never say never again. The NIMA-related protein kinases in mitotic control. Trends Cell Biol 2003; 13:221-228.

13 Lou Y, Yao J, Zereshki A, et al. NEK2A interacts with MAD1 and possibly functions as a novel integrator of the spindle checkpoint signaling. J Biol Chem 2004; 279:20049-20057.

14 Zhou R, Cao X, Watson C, et al. Characterization of protein kinase A-mediated phosphorylation of ezrin in gastric parietal cell activation. J Biol Chem 2003; 278:35651-35659.

15 Yao X, Anderson KL, Cleveland DW. The microtubule-dependent motor centromere-associated protein E (CENP-E) is an integral component of kinetochore corona fibers that link centromeres to spindle microtubules. J Cell Biol 1997; 139:435-447.

16 Xue Y, Zhou F, Lu H, Chen G, Yao X. GPS: a comprehensive www server for phosphorylation sites prediction. Nucl Acids Res 2005; 33:W184-W187.

$17 \mathrm{Fu} \mathrm{G}$, Hua S, Ward T, et al. D-box is required for the degradation of human Shugoshin and chromosome alignment. Biochem Biophy Res Commun 2007; 357:672-678.

18 Tang Z, Song Y, Harley SE, et al. Human Bub1 protects centromeric sister-chromatid cohesion through Shugoshin during mitosis. Proc Natl Acad Sci USA 2004; 101:18012-18017.

19 Waizenegger IC, Hauf S, Meinke A, Peters JM. Two distinct pathways remove mammalian cohesin from chromosome arms in prophase and from centromeres in anaphase. Cell 2005; 103:399410.

20 Church K, Lin HP. Kinetochore microtubules and chromosome movement during prometaphase in Drosphila melanogaster spermatocytes studied in life and with the electron microscope. Chromosoma 1985; 92:273-282.

21 Ault JG, Nicklas RB. Tension, microtubule rearrangements, and the proper distribution of chromosomes in mitosis. Chromosoma 1989; 98:33-39.

(Supplementary Information is linked to the online version of the paper on the Cell Research website.) 